\title{
THE TREATMENT OF ADVANCED CANCER
}

By Robert Cox, M.B.E., F.R.C.S.

Assistant Surgeon, Westminster Hospital

Any medical man who has had much to do with patients suffering from malignant disease must have had the experience of seeing a person whom he considered doomed to an early death from cancer live on for several years, often enjoying a useful and rewarding life, and this despite the fact that he may have widespread metastatic deposits, that he is in fact an ' advanced case of cancer.' Sometimes such episodes are inexplicable, sometimes they can be attributed to skilful management allied to a high resistance to the disease. The unpredictable way in which such cases occur is but one of the fascinations of this puzzling disease, and gives encouragement, should that be necessary, to a study of the management of what may be termed the advanced case.

It is necessary to be clear as to what we mean by this term 'the advanced case' and for the purpose of the present discussion it will be taken to mean those cases of malignant disease which by reason either of distant metastases or of extensive local spread cannot be dealt with by the routine and classical operations or by other conventional methods.

It is not the purpose of this article to consider the terminal phase of the patient's illness and the approach of death, though this is a matter which every practitioner should ponder, perhaps more than he does, since at this time, the patient still needs his doctor's help and support. It may, however, be appropriate to recall what two great surgeons said about approaching death. Treves had this to say: " The dread of death is an instinct common to all humanity. . . . Its counterpart is the instinct to self preservation, the resolve to live. It is not concerned with the question of physical pain or distress but is the fear of extinction, a dread of leaving the world. Quite apart from this natural and instinctive attitude of mind there is with many a poignant fear of death itself, of the terror and suffering that may be thereby involved. This fear is ill founded. What is termed the " agony of death" concerns the watcher by the bedside rather than the being who is the subject of pity. A last illness may be long, wearying and painful, but the closing moments of it are, as a rule, free from suffering. Quite commonly the actual instant of death is precede $\$$ by total unconsciousness. In other instances state of semi-consciousness exists up to almost the last moment of life. It is a dreamy condition free of all anxiety. Could the individual returrpo to life again no recollection of the period would I think, survive. It is a condition wholly free from uneasiness and from any suspicion of alarm, but is one suggestive of content.' The dying words of William Hunter were, we are told $\frac{\mathbb{S}}{}$ 'If I had strength to hold a pen I would write्్ how easy and pleasant a thing it is to die.' .

The management of a patient with cancer, ofece he has entered into the category ' advanced' nof lie along any or several of the following possibile lines of treatment.

I. Extended surgery and palliative surgery.

2. Radiotherapy.

3. Hormones and surgery of the endocrines in hormone dependent tumours.

4. Chemotherapy.

5. Relief of pain.

6. General regime.

The subject is so vast that one can only consider. each method in principle with some examples to amplify the views put forward. There is, however. one concept that must never be lost sight of and it is well put by Sainsbury, quoted by Gavey in his 'Management of the Hopeless Case.' H\& writes: 'To what end then this formidable array of remedies? To the relief of pain or of disease, generally, in the literal meaning of the word: to the prolongation of life. . . . Even then. if we are able to relieve distress there is still roort for us, but if this may not be, if, on the contrary, our efforts to eke out existence do but lengthen hopeless struggle, fretting instead of bringing comfort, then it will be for us to remember that the obligation to protract life at any cost is not laid upon us. In this view of our duty we shaB be strengthened if we bear in mind that the sting of Death is in the foretaste, in the anticipationg rather than in the realisation.' 


\section{Surgery}

The requirements for a 'good cancer operation' have long been recognised. They are the wide excision of the primary growth, together with as much surrounding tissue as is practically possible, and the immediate lymphatic drainage area en bloc. This is not, of course, attainable in all sites for reasons of anatomy and of the extent of such removal.

It is necessary here to clarify our ideas and terminology in regard to the words 'operable' and 'inoperable,' ' resectable' and 'unresectable.' A growth is said to be operable when the organ in which it arises can be removed together with any lymph nodes to which there may be apparent evidence of spread. If there is spread of disease beyond these limits, the lesion is said to be inoperable even though it is a simple matter to remove the primary growth itself. In these circumstances the lesion may be said to be resectable. A growth is unresectable when it is physically impossible to cut it out.

What is 'resectable' for one surgeon may not be so to another by reason of difference of training, experience, outlook and even of courage. But the gradual development and improvement of surgical methods and techniques and of supportive measures have brought more and more cases previously considered unresectable within the scope of the surgeon. It would be wrong, however, to leave without comment an implication that bigger and wider operations for cancer are thereby necessarily better operations. A case in point is carcinoma of the stomach. Increasing experience with total radical gastrectomy has led to increasing disappointment with results, the expected improvement in long term survivals has not been substantiated and many surgeons have therefore reverted to partial gastrectomy. On the other hand, all surgeons are agreed that where possible, a stomach which is the site of cancer should be resected even though it is inoperable, say by reason of glandular or hepatic spread or even peritoneal dissemination, the reason of course being that many of the patient's symptoms will be relieved by the removal of the primary growth. Moreover the removal of any large tumour is always a desirable end in itself, since it may be the source of malaise and other ill-defined symptoms due to toxic absorption, particularly if it is an ulcerated lesion. Sometimes when operation is undertaken the symptoms of which the patient complains may be of no great severity, for instance a little rectal bleeding which leads to the diagnosis of a carcinoma of the rectum. It is all too common in these patients at laparotomy to find extensive hepatic metastases which bring the case into the category of inoperable. Nevertheless, the lesion should be resected, since if left, the patient will later inevitably develop more severe and unpleasant symptoms such as pain and tenesmus. When this stage is reached it may then be too late to attempt any local removal, the lesion may be by then quite unresectable.

The fact that a growth is inoperable though resectable should not however go unconsidered and may suggest a modification of the procedure actually to be performed. Again quoting carcinoma of the rectum as an example, in these circumstances a case can be made out for doing a resection with re-establishment of continuity rather than a full abdomino-perineal resection, thereby saving the patient from the necessity of a colostomy. In such circumstances the surgeon must be reasonably sure that he is not running the risk of his patient developing an early local recurrence, whereby the advantages to be gained by conserving the sphincteric mechanism might be more than offset by a return of local symptoms.

One further aspect of this principle of resecting inoperable growths where possible must be mentioned. It is that sometimes when this has been done, there appears to be arrest of the growth of metastases or even in rare circumstances actual regression and this state of affairs may continue for a surprisingly long time, even years. This is a phenomenon which is also to be observed in the radiotherapy of tumours, notably of the reticuloses, when the treatment of a mass in one part of the body is sometimes followed by the spontaneous regression of lesions at a distance in other areas.

Inoperability may result not only from distant metastases but strictly also ensues when other contiguous organs are involved, e.g. carcinoma of the stomach involving colon and small intestine by direct spread. Ten years ago such patients were generally regarded not only as inoperable but also as unresectable and indeed such an opinion is all too easily accepted in some quarters even today, but advances in preoperative preparation, supportive measures such as blood transfusion and improved anaesthesia and postoperative management have brought the multivisceral resections required for such cases into the category of reasonable procedures no longer to be classified as heroic or desperate. Such operations demand foresight in preparation, determination and some experience in execution, and a patient who is not too senile and decrepit, but given these requisites are reasonable and fully justified. I know of one patient who is alive and well five years after the removal of his transverse colon, the lower two thirds of his stomach, a loop of small intestine and an area of anterior abdominal musculature about the size of a large saucer, all involved by direct extension from a primary in 
the colon. The gap in the parietal muscles was made good with a sheet of tantalum wire gauze beneath the skin and the anxieties expressed that it might ulcerate into the intestine, cause ileus or in some other way misbehave have all proved groundless.

Many years ago Grey Turner urged that involvement of the abdominal parietes should in itself never preclude a serious and determined attempt to extirpate the lesion. Even if the gap needs to be of full thickness and including skin, it can, with the use both of ingenuity and sometimes vaseline gauze, be closed.

Most of these multivisceral resections have to be done secundum artem as the exact details of the spread of the lesion cannot be known until the abdomen is open. In a rather different category are those operations done for extensive cancer of the pelvic viscera and often associated with the name of Brunschwig. In these cases the primary growth is most commonly situated in the cervix uteri with local extensions into the bladder, urethra, ureters, or the rectum, so that no operation short of a total clearance of the pelvic contents can possibly hope to cure or effectively palliate the disease. Carcinoma of the vagina, of the bladder with posterior extension, or of the rectum with anterior extension may raise similar problems in which all three tracts, urinary, genital and alimentary will have to be in some part sacrificed. There are, however, cases where the extension may not be so great and where a less formidable operation may suffice, i.e. resection of bladder and genital tract or of rectum and genital tract.

The operation of pelvic viscerectomy is now well established and when properly performed in a suitable patient is capable of giving great satisfaction. Surprisingly, the patients are usually able to lead a happy and useful life. I have one patient who is over sixty and works as a barmaid in a well-known London tavern, while another of similar age survived this large procedure six weeks after suffering a major coronary infarction.

It should be clearly understood that the total operation is much easier to perform than either of the more limited operations in which bladder or rectum is preserved. If the patient is placed so that a combined synchronous abdomino-perineal approach can be made by two surgeons and the internal iliac arteries are tied early in the operation, usually just after the ureters have been identified and isolated, there is seldom any great technical difficulty in the actual removal. It is possible in most cases to isolate a small length of the sigmoid colon on its own vascular pedicle and after closing one end to bring the other out onto the abdo'minal wall, where it is sutured. Into this short isolated piece of colon, the ureters are im- planted so that the urinary stream is kept separate from the faecal stream. (Cade, r954). The patient then has a colostomy which can be managed in the standard way and which, with any luck, $c$ will work once a day, and a ' urinostomy' which drains into a 'sticky' bag of the Rutzen type. This is a vastly better state of affairs and far more $\overline{0}$ hygienic than a "wet' colostomy.

It is important in considering patients for $\frac{\overline{\bar{p}}}{\bar{\phi}}$ this operation to select those who are psycho- $\mathbb{D}$ logically suitable and have sufficient adaptability and determination to face up to their new $\overrightarrow{0}$ ' arrangements.'

When the operation is first proposed-and one $\overrightarrow{\vec{\omega}}$ must be frank in doing this-the patient is often $\frac{\text { s }}{\delta}$ appalled at the prospect and will not believe that $\frac{0}{3}$ life afterwards can be either tolerable or worth while. The help of a previous patient who has $\underset{\perp}{\omega}$ made a success of it and who is willing to visit the $\omega_{\infty}$ hospital and talk to the sufferer is quite invaluable on these occasions.

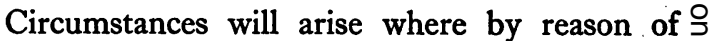
spread of disease or the frailty of the patient such operations as the foregoing are not practical. It $\mathbb{\oplus}$ may then be that the surgeon can only wait on events until such time as the patient's condition and symptoms dictate that a palliative colostoms or cystostomy are indicated. Such palliative ' ostomies' are best delayed, since they are ofte a source of tribulation in themselves and relievs the patient only in part of their most trying symptoms. One such operation much practised in the past, gastrostomy for carcinoma of the oesophagus, is in fact to be condemned as it does nothing to $\mathbb{D}$ relieve the patient of his symptoms and merely $\vec{F}$ keeps him alive the longer to endure the distress of constant coughing and inability to swallow his own saliva. Where possible, by-pass operations or the introduction of a Souttars tube are infinitely to be preferred for the palliation of patients with unresectable cancer of the gullet.

When distant metastases have occurred, surgical extirpation is not generally considered to be appropriate, but there are two special cases where this rule may be ignored with considerable chance of benefit to the patient.

Hypernephroma at times produces solitary $\stackrel{\frac{D}{0}}{=}$ distant metastases which are resectable and $N$ sufficient examples of removal of such lesions with survival of several years are recorded to make $\triangle$ consideration of such a course well worth while. $\omega$ In Highams case, for instance, a patient had his kidney removed for a hypernephroma: nearly two years later he had a lobectomy for a pulmonary $\mathbb{D}$ metastasis and after the passage" of yet another? one and a half years developed symptoms of a cerebral tumour. This was operated on and 
proved to be another 'solitary' metastasis, the patient surviving for a further three years.

The other disease in which the natural history dictates special consideration is malignant melanoma. In this instance the course of the disease is not always as rapid and overwhelming as is popularly thought, and where resectable metastases occur-as opposed to widespread dissemination-they should be resolutely tackled, since if left they will ulcerate and tend to accelerate the course of the disease. Again such a policy will often pay handsome dividends in prolonged and more comfortable survival.

To sum up then, the place of surgery is to remove resectable lesions since by so doing, the patient's condition will be made more comfortable, and where this is impracticable, to apply operations of diversion in order to mitigate pain and misery. To select what is practical and what helps towards the patient's longer survival in reasonable comfort, however, calls for both experience and judgement.

\section{Radiotherapy}

Where malignant lesions are for some reason unresectable, or where the patient refuses operation, radiotherapy is an obvious choice in an attempt to help. But there are instances where even though surgery could be employed, radio therapy should still be the first choice. Cases of carcinoma of the breast, which are associated with considerable enlargement or early fixation of the axillary glands, i.e. late Stage II, or where there is considerable peau d'orange or skin infiltration will be probably made worse by surgery and should be treated in the first instance by radiotherapy.

Radiotherapy as delivered by conventional methods, i.e. X-rays generated at 250 or $400 \mathrm{Kv}$. or gamma rays derived from teleradium or telecobalt units, is usually only of use in epidermoid cancer (skin, mouth, tongue, pharynx, oesophagus and lower genital tract), cancer of the breast, the reticuloses and some other special sites. Evidence is accumulating, however, that with increasing voltages and energy outputs, $\mathrm{X}$-rays will in some cases prove to have a considerable beneficial effect even on adenocarcinoma of intestinal origin, generally believed in the past to be totally radioresistant. But to be of use in this way the voltage at which the $\mathrm{X}$-rays are produced must be of the order of two million or greater. Another advantage of such super voltage irradiation is that the unpleasant side effects (skin reactions, malaise, nausea) are greatly reduced or even absent, while the tumour dose delivered is very much higher than with conventional X-rays. Also the length of treatment times are shorter so that the advantages to an ill patient are considerable.

It must be remembered that as with surgery, it is possible to differentiate between radiotherapy which is attempting to be curative and that which is clearly recognized to be only palliative in intention. In the latter case the dose employed is lower and the whole technique less rigorous from the patient's point of view. Where the patient is clearly incurable, there is no point in inflicting too severe a treatment, in fact definite harm may result in that the patient's resistance is lowered and other metastases may be encouraged or accelerated.

The site and extent of metastases are all important in deciding the applicability of radiotherapy. Limits are imposed by the total tissue volume exposed and an integral dose is recognized beyond which it is not safe to go. Moreover, lesions so situated that spleen, liver and kidneys are in the beam of radiation will result in damage to these structures which may more than offset any possible advantage.

Pulmonary metastases, since they are so common, require separate consideration: they are often symptomless until quite late on and even though they are derived from a sensitive tumour are best left untreated by irradiation. The lung parenchyma is itself very liable to damage by $\mathrm{X}$-rays and post radiation fibrosis can be rapid in onset and most crippling in its effects. These remarks apply to widespread pulmonary deposits but where one single metastasis is found, then radiotherapy is a more reasonable proposition.

$\mathrm{X}$-ray therapy may also be used palliatively to effect no more than the control of some distressing or troublesome symptom such as haematuria from a growth in the urinary tract. In a similar way pain arising from malignant deposits, particularly in bones, may be controlled even if only temporarily and of course without modifying appreciably the general course of the disease.

Pathological fractures provide another instance where considerable help is to be had from the proper use of palliative radiotherapy.

In addition to the techniques indicated above, a well-equipped and modern radiotherapy department has available radio active isotopes, some of which can be helpful in the management of certain cancer patients.

Of these isotopes radio active Iodine (I. I $3 I$ ) is the one which is capable of producing the most striking effect when applied to cases of cancer of the thyroid with metastases. Certain points are important in such cases. First, all the thyroid gland itself must be completely extirpated or the iodine will be taken up there. Secondly, it is necessary to show by means of a tracer dose that 
the tumour is of the type that will pick up and utilize the iodine and thirdly the therapeutic dose of radio active iodine must be chosen with some circumspection. When the iodine is taken up in the metastasis, the latter is of course converted as it were into a radioactive source which, if too large a dose has been given, may be so powerful as to inflict unpleasant and unnecessary symptoms on the patient. It is unfortunate that by no means all cases of cancer of the thyroid with metastases are susceptible to treatment by radio active iodine. In fact only the minority, probably less than ro per cent., will respond in any way and it is generally said most of these will be well differentiated alveolar adenocarcinomas.

Phosphorus (P. 32) is another isotope which has been used and has given some help in the treatment of the leukaemias, but not to the extent that was hoped when it first became available.

Solutions of radioactive gold salts (Au. I98) are also employed but are applied locally by injection into either the pleural or peritoneal cavity in cases where those serous membranes may be the site of malignant deposits with consequent blood stained effusions. Using such a technique it is possible to reduce the rate at which the cavity refills after tapping and thereby render the patient more comfortable for longer periods.

\section{Hormone Dependent Tumours}

In the whole field of malignant disease there can be no more striking instance of the development of new concepts of disease control than in the case of what Huggins has called the ' dependent tumours,' i.e. carcinoma of the breast and prostate.

Although some connection between the gonads and the course of carcinoma of the breast was recognized as long ago as 1896 by Sir George Beatson, it is only since the introduction of synthetic oestrogens and androgens that widespread interest has been aroused, and the later availability of cortisone and its related steroids has both stimulated research into these cancers and utterly transformed the immediate prospect for many patients with advanced disease.

Some of the results of hormone therapy are so remarkable that there is perhaps a temptation to resort to this item of therapy too early or too readily. Moreover the role of hormones and endocrine surgery vary considerably in cancer of the breast and prostate and it will be necessary to consider each disease separately.

\section{Cancer of the Breast}

Since the progress of this disease can be retarded by surgery, by radiotherapy and by endocrine methods, the question is sometimes asked why not combine all three methods and apply them at the same time, on the principle of maximal concentration producing maximal effect in an attempt completely to erradicate the disease. There is in fact no evidence to support the belief that such a combination will in fact have the effect desired and the present position is that one should use all the methods available in turn or at intervals, so as to prolong the period of control to the maximum.

When a case of carcinoma of the breast has passed beyond the stage where the disease is localised, radiotherapy should still be considered as the first weapon to be employed, especially where the problem is lymphatic spread such as involvement of supraclavicular or contra lateral lateral axillary nodes. The same applies where bone metastases have occurred, so long as these are not too widespread, thereby presenting a target too extensive for practical management by radiotherapy.

The radiotherapist's aim at this stage should be to control the disease rather than to try and cure it and he should use the minimum dosage to gain this objective. In this way unpleasant and debilitating reactions can be avoided and skin damage minimised so that a second course of treatment, where called for, can be given.

By judicious therapy of this type it is possible to have some patients exist for a number of yearso living with their disease quite comfortably.

Sooner or later, of course, there comes a time when X-rays will no longer control the disease or the dissemination becomes too widespread. It is now the turn for hormones to play their part and the method of employing them depends to a considerable extent on the patient's age.

In the younger group, that is to say the premenopausal and those who have completed their menopause in the last five years, androgens should be given. In the youngest patients who have many years to run before the menopause can be expected, oophorectomy should be seriously considered at this stage, especially if the response to androgen therapy gives ground for believing the case to be hormone dependent. Unless there is some very good reason the ovaries should be removed by operation, and the temptation offered by an X-ray menopause should be resisted, since the completeness of the latter in terms of hormonal output is doutbful to say the least.

An important point arises in regard to the method of exhibiting the androgens. Experience shows that oral administration is far less satisfactory than injection, both as regards the disease which is less powerfully affected and as regards the patient who suffers more from the unpl easant side effects such as hirsutism and virilism.

Testosterone proprionate in doses of $150 \mathrm{mgm}$. 
should therefore be given twice a week until such time as the patient has had a minimum of 3,000 mgm. when a rest may be given and then the drug subsequently resumed as soon as there is the slightest sign, either subjective or objective, of renewed activity of the disease.

There are, apart from considerations of age, certain patients where testosterone is contraindicated or only to be used with great caution: these are patients with:

I. A raised blood calcium, particularly if associated with

2. A poor renal function and

3. Ascites or hydrothorax.

If any of these conditions are present the energetic use of androgens may precipitate a fatal outcome and such patients will have to be considered at once for adrenalectomy or some other method of control.

Androgen therapy will, if it is going to be successful, often produce an improvement of which the patient is aware in so short a period as a week, though the full effect will take much longer to develop. The results can be truly reremarkable, especially in the regression of large visceral metastases and the healing and recalcification of boney deposits.

Where the patient is aged, say over 65 , it is wise to try the effects of oestrogen therapy, as androgens are likely to be ineffective or may actually be harmful. The oestrogens can be given in any form, but Stilboestrol serves very well and quite small doses only are required, no more than $5 \mathrm{mgm}$. being given twice a day. Larger doses will be no more effective in modifying the disease and will only cause uterine haemorrhage with consequent alarm and distress to the patient.

There is in addition, a group of patients between about 55 and 65 years of age who do not fall easily into either category. These, when necessary, are best started on androgens, but rather more cautiously than the younger patients and should deterioration occur, the policy can be reversed and oestrogens substituted.

Whichever hormone is being used, sooner or later it will fail to control the disease and the time has then arrived to consider whether a surgical attack should be made on the endocrines. At this stage it is necessary to draw attention to the fact that all cancers of the breast are not hormone dependent. The figure varies in different series but somewhere in the neighbourhood of 60 per cent. of tumours is about the maximum which one can hope to modify favourably by these methods. If there has been a response, either favourable or unfavourable, to hormone therapy, then one can expect some result from surgical attack on the endocrines. The method most widely applicable is bilateral adrenalectomy, though hypophysectomy must also be considered. More is known about adrenalectomy and it has the obvious advantage that it is a procedure within the scope of any general surgeon, whereas hypophysectomy requires the special operative technique possessed usually only by the trained neurosurgeon. Hypophysectomy has the further disadvantages that it is not applicable where there are cranial or intracranial metastases, that there is some risk, albeit small, to vision and finally many of the patients have a troublesome diabetes insipidus following the operation. On the other hand, in the only se ries where an attempt has been made to compare the two operations there is some evidence to suggest that there may be slight advantage accruing to hypophysectomy and this operation is still available to the patients who by reason of extensive involvement of their retroperitoneal tissues are beyond adrenalectomy, a state of affairs which is usually only revealed when the operation is attempted. Hypophysectomy is also worth considering when a patient has already had an adrenalectomy with benefit, but has relapsed.

Where adrenalectomy is to be performed in a patient of the younger age groups, it should be combined with oophorectomy if the ovaries have not already been removed. A common practice is to remove the ovaries and the left suprarenal at the first operation and to take the right adrenal at a second operation a week or ten days later. $A$. remarkable feature of these patients is the way in which, in many cases, pain is relieved to some extent even after the first operation, and the relief being increased, of course, after the second side is done.

Such patients, of course, have to be given cortisone before operation and have to continue taking the drug permanently after operation. Any subsequent operation or acute illness has to be covered by an increase in the daily amount of cortisone. Illnesses where vomiting or diarrhoea, particularly the former, occur are particularly dangerous and often require the readmission of the patient to hospital so that disturbances of electrolyte balance can be corrected and cortico-steroids given parenterally.

The question may arise as to what to do with the patient who has shown no response to androgens. Such patients are clearly less likely to respond to adrenalectomy but nevertheless should be given the chance unless desperately ill. Where these circumstances obtain or the patient is deemed for other reasons unsuitable for operation or refuses surgery, suppression of adrenal cortical activity may be attempted by giving cortisone or preferably Prednisone. This latter preparation has the advantage that it tends to cause less water 
retention than cortisone and when given in ro mgm. doses twice daily, may have a very beneficial effect on the course of the disease.

Reference must be made to radiotherapeutic ablation of the pituitary. This was first attempted by introducing radon seeds through a trocar and canula passed via the nose through the floor of the sella turcica into the pituitary. Many unfavourable results and some favourable were reported. Among the unfavourable results were blindness due to damage to the optic nerve by radiation, cerebrospinal rinorrhoea and meningitis and this led to the substitution of gold seeds for radon and later still small rods of radio active ytrium. The incidence of complications and disasters has been reduced in this way but at best this is a method with a limited usefulness.

\section{Carcinoma of the Prostrate}

Carcinoma of the prostrate is the other disease which is hormonally dependent. It differs from cancer of the breast in being much less common, occurring in an older age group with a small age range, being relatively uncommon before the age of 60 and lastly being on the whole more uniformly sensitive to hormone control. Yet another difference is that radiotherapy plays little part in the treatment of this disease, though painful bone metastases may respond well to $\mathrm{X}$-rays. The vast majority of the cases, however, are well controlled for several years on oestrogens alone, though there is an increasing body of opinion which favours subcapsular orchidectomy as soon as the diagnosis is confirmed either by histology or by the radiological evidence of bone metastases. Subcapsular orchidectomy is a small operation and may in itself be sufficient to control the disease, but where this is not so it will result in potentiation of the effect of such oestrogens as are used.

It follows from what has been said that prostatic candidates for adrenalectomy are few, and for hypophysectomy even fewer and it is difficult to give an authoritative statement as to results. It would seem, however, that the results of major endocrine surgery in these patients can be as dramatic as in the breast cancer patients but the favourable response is apt to be of the order of six to nine months as against 18 months or two years for breast cases.

A rather special problem is the male patient with carcinoma of the breast. Of whatever age he may be, adrogens are unlikely to help since he probably has already an ample supply; likewise oestrogens are contra-indicated since the wellknown effect on the male breast of this drug is not likely to be anything but harmful. One is left, therefore, with orchidectomy as the only reason- able method of endocrine control and if and whep this fails, resort must be had to adrenalectomy or or hypophysectomy.

\section{Chemotherapy}

The use of oestrogens to control cancer of the prostate may be said to have marked the beginning of the era of medical or pharmacological treatment of cancer, a concept which must always appeal to anyone who has to treat this disease, especially in its late stage with widespread dissemination.

Oestrogens, androgens and cortico steroids are limited in their effects to cancers of sex organs. They have no effect on all the other forms of malignant disease.

The observation of a profound and persistent leucopenia in men poisoned by mustard gas led to research and the development of compounds known as nitrogen mustards, which are of great use in the treatment of the reticuloses. Here again as a general rule the primary treatment should be radiotherapy but as the disease progresses and one course of X-rays follows another, each seems to become less effective. It is at this stage that the nitrogen mustard treatment is invaluable. These cytotoxins have, of course, an unpleasant effect on the patient as is to be expected from cell poisons, and since they are only effective in a proportion of $\varnothing$ reticuloses other chemotherapeutic agents have been sought and found. Among these are substances which are of the anti folic acid type and? those which interfere with the action of ribo nucleic acid in the metabolism of the chromatin of the nucleus. The selection and employment of these chemotherapeutic agents has become a speciality in itself and is outside the scope of the present article.

\section{Relief of Pain}

Some patients with advanced malignant disease pass through the whole course of their disease without suffering pain and in many, though discomfort and distress may be well marked, pain is a relatively minor trial. Unhappily there are those in whom pain may be not only severe but also prolonged and where this occurs in persons who lack both strength and stoicism, the result may be most distressing to all concerned.

Pain may be relieved or controlled by drugs or by anaesthetic and surgical procedures.

As a general principle the minimum dose of the simplest drugs should be used at first and it is surprising how long the patient may be kept in reasonable comfort by no more powerful agent than aspirin, perhaps allied with phenacetin and codeine. A very useful mixture which will control any but the severest pain is one containing aspirin and tincture of opium or nepenthe, suitably 
flavoured with chloroform water. The amount of opiate is insufficient to cloud perception or dull the faculties and the main drawback to its use, as with codeine, is the constipating effect on the patient. Physeptone, pethidine and such like drugs are best avoided since despite all claims to the contrary, they are habit forming and the patient comes to desire them even when the pain is not troublesome and therefore increasing tolerance and the demand for bigger dosage is developed. The same applies to any of the narcotic analgesic drugs and eventually toxaemia, increasing debility and breakdown of personality result from these drugs. This deterioration in the patient's physique and morale can lead to greatly increased distress among his friends and family, particularly if there yet remains a considerable period to endure, or if the malignant disease is taking its toll more slowly than was at first anticipated. This is a danger which is all too seldom appreciated by those who have the difficult task of caring medically for patients who are to pass the final stages of their disease in their own homes, and a timely warning not to resort too readily or too early to the giving of powerful narcotic drugs may save much unhappiness to all concerned.

There are, of course, many patients in whom the pain occasioned by their disease and sometimes also in part by their treatment (i.e. radiation) may be most severe. Where this state of affairs exists in a patient who is physically still in good condition and where the disease may yet be expected to run a fairly long course, resort to some form of nerve interruption should be earnestly considered.

The methods available are:-

I. Nerve blocking.

2. Nerve or nerve root section.

3. Spinal tractotomy.

4. Leucotomy.

Nerve blocking using local anaesthetics will, of course, give relief of only short duration, but is useful to explore the possibilities of relief, after which some longer acting agent such as an oily anaesthetic solution or phenol may be employed. Ethyl alcohol probably gives the most complete and longest maintained interruption of nerve conduction and the risk of the subsequent development of a painful neuritis is not so serious in these patients who have, in any case, a limited survival.

Nerve blocks are most useful in the trunk, since when applied to the limbs, all sensation, tactile, temperature and positional will be lost. Blocking of a peripheral mixed nerve also brings about paralysis which perhaps in the circumstances of the disease may not be important, but can be avoided if necessary by subarachnoid blocking of the posterior nerve roots as described by Bonica, who gives an excellent account of the whole subject in his book ' The Management of Pain.'

Nerve blocking for trunk or visceral pain will almost certainly require to be applied not only to somatic nerves but also to the sympathetic nerves which carry many of the painful afferents from the visceral area.

One of the great advantages of nerve blocking is that it can be used in debilitated patients whose physical reserves are low-on the other hand this technique has the disadvantage that it may fail to give complete relief.

Therefore in patients who are still strong and require some relief of this kind, rhizotomy or cordotomy may be more likely to meet the requirements of the case. It must be realised, however, that both these operations are major procedures requiring a laminectomy and carry a definite mortality and morbidity rate, while 100 per cent. success in relieving pain cannot be promised.

Rhizo tomy, like nerve blocking, has the disadvantage that it destroys all senstation, touch, temperature and position as well as pain and this may not be acceptable. Antero-lateral cordotomy (division of the spinothalmic tracts) overcomes this difficulty since only pain and temperature fibres are cut, but has the disadvantage that sphincter control may be interfered with, either temporarily or permanently and when done bilaterally is almost invariably accompanied by some weakness in the lower limbs. This, however, may not be of such great consequence in pelvic visceral malignancy where the sphincteric actions may already be compromised or destroyed and the patient partly bedridden. In such patients, the results of cordotomy may be extremely gratifying, but even in the most skilful hands, there is a definite mortality, Olivecrona for instance giving a figure of 12 per cent.

Lastly, prefrontal leucotomy has been used in the control of pain. This operation does not abolish pain: it alters the patient's reaction to it so that they are no longer distressed or upset but at the same time, changes of personality are liable to take place and these are quite unpredictable. These personality changes may be profound and unpleasant, occasioning great distress to the patient's friends and relatives. It is therefore a method which has gained little favour and is not much practised in this country.

Finally, much can be done-or fail to be donefor these patients in the way of general medical regime and nursing care. The maintenance of nutrition, correction of anaemia, attention to bowels, control of urinary infection where present, proper care and dressing of fistulae, ulcers or pressure sores, the provision of an outside interest 
by suitable occupational therapy are just some of the points that will contribute to the ease and comfort of these patients in the closing stages of their disease. All of these points can be attended to and very often are in the patient's own home, but this will only be contrived if the medical man in charge continues to the last to take the keen, lively and sympathetic interest which his patient is entitled to expect.

\section{BIBLIOGRAPHY}

BEATSON, SIR G. T. (1896), Lancet ii, 104, I62.

BONICA, J. J. (1953), 'The Management of Pain,' Henry Kimpton, pp. 499 et seq.

CADE, SIR S. (1954), Annals Col. Surg., 15, pp. 71-107.

CADE, SIR S. (1954), Brit. Surg. Prog., p. 131.

GAVEY, C. J. (1950), 'The Management of the Hopeless Case,' H. K. Lewis \& Co. Ltd.

HIGHAM, A. R. C. (1953), Medical Press ccxxix, 5952.

OLIVECRONA, H. (1947), Acta psychiat. and neurol., 46, 268.

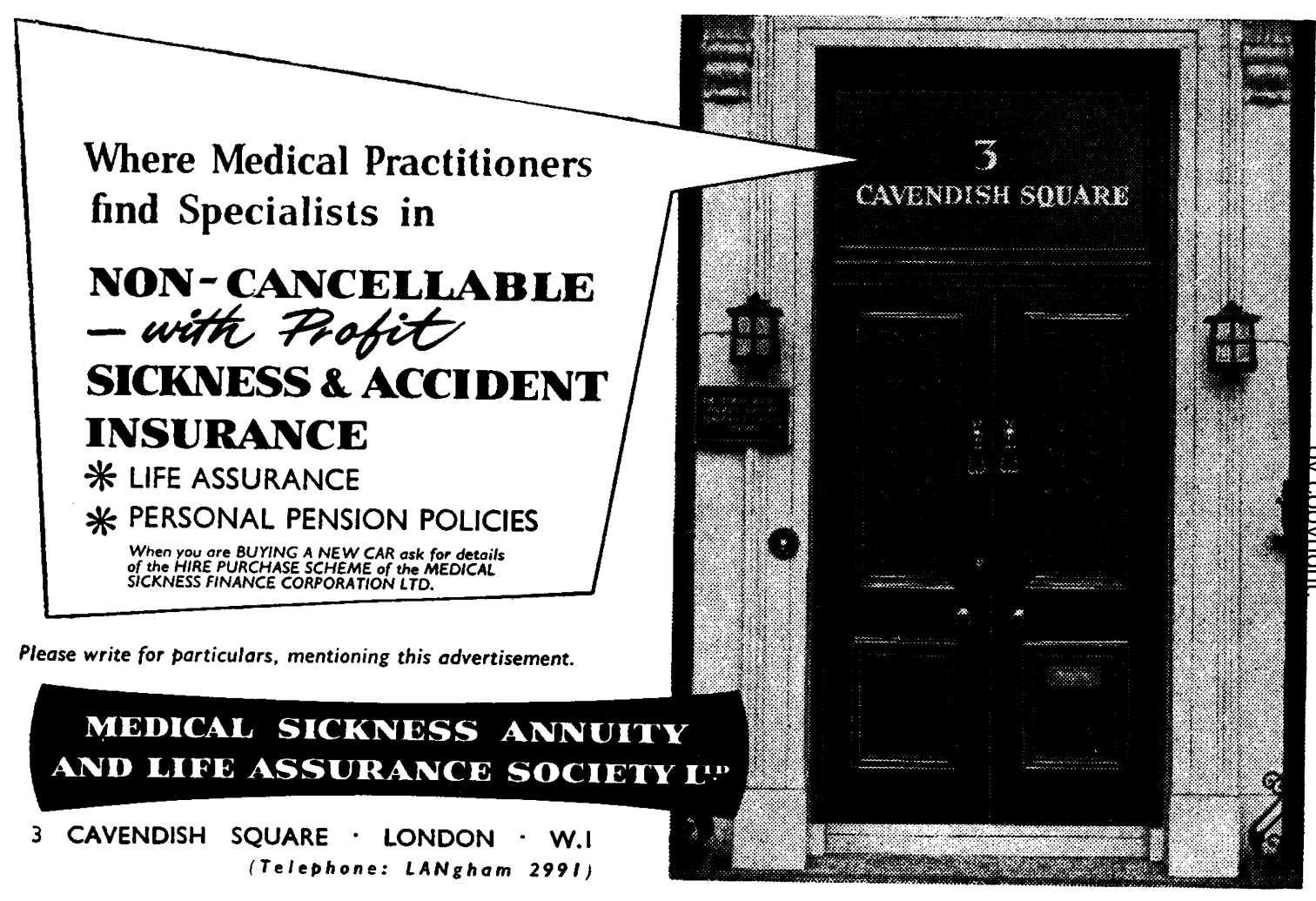

MEDICAY SICKNESS ANNUITY

AND LIFE ASSURANCE SOCIETY $L^{4 !}$

3 CAVENDISH SQUARE - LONDON - W.I

(Telephone: LANgham 2991

\section{NOTICE OF SPECIAL INTEREST TO SUBSCRIBERS: \\ -WHY NOT HAVE YOUR COPIES OF THIS JOURNAL BOUND INTO YEARLY VOLUMES?'}

You can have your twelve monthly issues fully bound in dark green pin head cloth. lettered in gilt on spine with name of Journal, Volume Number and year, complete with index at front, for $21 \mathrm{~s}$. Od. post free. A limited number of out of print journals are available to bind into volumes and make your library complete. Price on application giving details of issues required to complete back volumes.

THE FELLOWSHIP OF POSTGRADUATE MEDICINE 60 PORTLAND PLACE, LONDON, W.I

\section{WHY NOT HAVE YOUR JOURNALS BOUND?}

